\title{
On the Scaling of Non-Asymptotic Capacity in Multi-Access Networks with Bursty Traffic
}

\author{
Florin Ciucu \\ Deutsche Telekom Laboratories / TU Berlin \\ florin@net.t-labs.tu-berlin.de
}

\begin{abstract}
The practicality of available (throughput) capacity results in multi-access networks, which dispense with coding schemes, is often questioned for several reasons including 1) the underlying asymptotic regimes, and 2) the assumption of saturated traffic sources. This paper jointly addresses these limitations by providing capacity results in non-asymptotic regimes, i.e., holding at all time scales and network sizes, for the very broad class of exponentially bounded burstiness (EBB) traffic sources. Both upper and lower bounds on capacity are derived in terms of probability distributions, which immediately yield all the moments. The explicit and closed-form nature of the results enable the investigation of the impact of burstiness on non-asymptotic network capacity. In particular, the results show that for the EBB class the non-asymptotic end-to-end capacity rate decays linearly in the number of hops.
\end{abstract}

\section{INTRODUCTION}

Information theory has been instrumental to many technological advances, particularly in the field of communications. However, in what is referred to as an unconsummated union, information theory has yet to make a comparable mark in the field of communication networks [13]. Part of the reason is that the traditional information theory approach to the problem of multi-access communication considers the noise and interference aspects of multi-access communication, but ignores the fundamental aspects of data burstiness and delay in packet switching networks [15].

Multi-access communication is an important but largely open problem concerning the understanding of the fundamental performance limits of communication in multi-hop wireless networks. Metrics of interest include the network (throughput) capacity, referring to the maximal data rates which can be reliability sustained by the network, and network delay. The pure information theory approach to the network capacity problem produced solutions restricted to special network topologies [15]. Simplification of the problem, i.e., by dispensing with multi-user coding schemes as in the work of Gupta and Kumar [17], produced solutions for more general topologies but in asymptotic form. These results, although shedding light on how network capacity scales in the number of nodes, are unable to predict the exact capacity in networks with a given number of nodes, and are thus questioned on their practicality for small or medium sized networks ([2], p. 180). Besides their asymptotic nature, the capacity results from [17] are derived under saturated conditions (i.e., all nodes have infinite data to transmit) and may be thus inaccurate for realistic network scenarios with bursty data sources.
In this paper we address the problem of (non-asymptotic) network capacity under data burstiness constraints at the sources. Concretely, we consider a fixed multi-hop path in a network with a specified multi-access protocol and with the transmitting source having data variability or burstiness. In particular we consider the class of Exponentially Bounded Burstiness (EBB) [24] data sources which include many Markov arrival processes. The relaying nodes on the fixed multi-hop path have only the role of relaying the data from its source to destination, whereas all the other nodes in the network are saturated. The contribution of this paper consists in the derivation of closed-form explicit results on the capacity of the fixed multi-hop path, in terms of both upper and lower bounds, with EBB arrivals at its source.

Besides applying to a wide class of data sources, what makes our capacity results particularly attractive is that they are non-asymptotic, i.e., they hold for any finite time scale and network size, and that they are derived in distribution, i.e., moments of any order can be readily obtained. Being derived in closed-form (up to the evaluation of infimum operators), our results enable the study of the impact of the degree of burstiness on the end-to-end achievable capacity rates. Numerical results show that capacity is sensitive to burstiness, in the sense that burstiness at the data source eventually dominates the effects of multi-access. In a multi-node setting, the bounds show that non-asymptotic capacity rates decay linearly in the number of nodes for the EBB traffic class. An open and far from apparently trivial question is whether this scaling holds for other types of bursty traffic such as heavytailed, which may create subtle correlations possibly leading to super-linear decays; such decays have been recently observed in wired networks even for EBB sources [6].

The analytical tool which enables the analysis in this paper is the stochastic network calculus [7], [18]. This is a probabilistic extension of the deterministic network calculus conceived by Cruz in the early 1990's [12], and is being regarded as an alternative to the traditional queueing theory [19], especially for multi-node queueing networks scenarios. The stochastic network calculus is herein applied to account not only for queueing delay due to data burstiness, but also for the access delay due to multi-access. Using the fundamental result of a network service curve, which provides a description on the service capacity along a network path in an underlying $(\min ,+)$ algebra, the paper can derive concise non-asymptotic network capacity results, and yet in a rigorous manner. 
In the literature, throughput capacity results are mostly restricted to averages in single-hop scenarios with specific arrivals [1], [21]. In turn, multi-hop results often rely on simplifying technical assumptions [16], or they are obtained asymptotically and in saturated regimes [20], [17]. Multihop non-asymptotic results have been recently obtained for saturated data sources [10].

The remainder of the paper is organized as follows. In Section II we introduce the network model along with the main analytical tools to be used along. In Section III we derive the main results of the paper, i.e., upper and lower bounds on the end-to-end throughput capacity, and also illustrate them numerically. Brief conclusions are presented in Section IV.

\section{Model, Arrival Processes, And Service Curves FOR MULTI-ACCESS}

We consider a slotted time model with slots of size one time unit. Also, we consider the network from Figure 1 in which node 1 transmits to node $k+1$ using the nodes $2,3, \ldots, k$ as relays. There are $N$ nodes inside the interference ranges of each of the relay nodes, represented in the figure by circles; the actual number of nodes in the intersection of adjacent interference ranges is not needed in the model. All nodes inside any interference range share the same communication channel, with capacity of one data unit per time slot, and use the (slotted-)Aloha protocol for multi-access communication in a full-duplex mode. Each node attempts to access the channel with probability $p=\frac{1}{N}$ in each time slot, independently of the other nodes. A transmission from node $i$ to node $j$ is denoted by $[i \rightarrow j]$. A transmission $[i \rightarrow i+1]$ is considered successful in some time slot if node $i$ has data to transmit, attempts to access the channel during that slot, and all the other nodes within the interference range of node $i+1$ are silent.

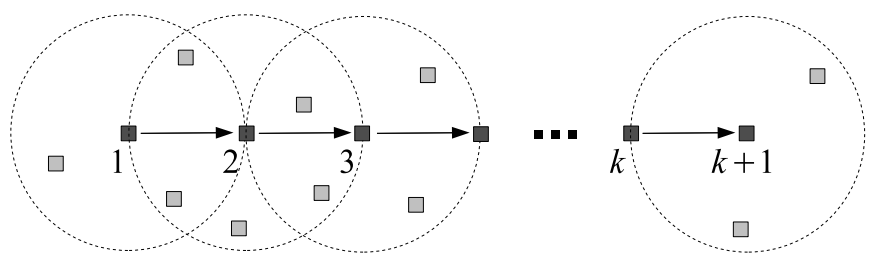

Fig. 1. A multi-hop wireless network with $k$ hops. The $k+1$ circles, representing the interference ranges of nodes $1,2, \ldots, k+1$, have each $N$ nodes inside, some of which are shared. We are interested in the (nonasymptotic) (throughput) capacity of node 1 transmitting to node $k+1$ using the nodes $2,3, \ldots, k$ as relays

The choice of positioning the relay nodes at the intersection of adjacent circles is simply made here for a more descriptive visual representation. In fact, our analysis can be readily extended to network models with different random number of nodes inside different circles, different transmission/interference ranges, or accounting for spatial correlations across more than two adjacent circles. The technical key enabling the rigorous analysis of such scenarios is the $(\min ,+)$ convolution representation of the end-to-end service.

In the following we first describe the arrival and departure processes' models, and then the $(\min ,+)$ service model.

\section{A. Arrival and departure processes}

We assume that all the nodes excepting $1,2, \ldots, k+1$ are saturated, that is they always have data to transmit to some (unspecified) nodes. The relay nodes only relay the data of the end-to-end transmission $[1 \rightarrow k+1]$. In turn, we assume that node 1 is fed by a data source with an arrival pattern characterized by burstiness or high variability.

We point out that the saturation assumption leads to a worstcase analysis for the end-to-end transmission $[1 \rightarrow k+1]$. Significant technical difficulties would arise, if this assumption were relaxed, due to subtle correlations amongst temporarily saturated/unsaturated nodes (for a related discussion on Aloha stability see [4]). One may relax in turn the assumption on the relay nodes, i.e., they may produce their own data or relay data for other transmissions but $[1 \rightarrow k+1]$, and run various scheduling algorithms (see [10]).

To model the arrival traffic burstiness at node 1 we consider the class of EBB processes [24]. These processes include many Markov arrival processes and can be described in terms of bounds on their moment generating functions (MGF's) [7], [14]. Let us denote $A(t)$ as the cumulative arrival process at node 1 which counts the total number of arrivals by time slot $t$, starting with $A(0)=0$. The EBB characterization assumes that for any $\theta>0$ there exists a rate $r^{U}$, depending on $\theta$ and invariant to time parameters, such that for all $s \leq t$

$$
E\left[e^{\theta A(s, t)}\right] \leq e^{\theta r^{U}(t-s)},
$$

where $A(s, t):=A(t)-A(s)$ denotes the corresponding bivariate process. This description is equivalent, up to a prefactor, to the original EBB description from [24]. We also assume the existence of bounds on the Laplace transform of $A(t)$, i.e., for any $\theta>0$ there exists a rate $r^{L}$, depending on $\theta$ and invariant to time parameters, such that for all $s \leq t$

$$
E\left[e^{-\theta A(s, t)}\right] \leq e^{-\theta r^{L}(t-s)} .
$$

An immediate consequence of Jensen's inequality is that for all $t>0$

$$
r^{L} \leq \frac{E[A(t)]}{t} \leq r^{U},
$$

which implies that the long-term average rate of the process $r:=\lim _{t \rightarrow \infty} \frac{A(t)}{t}$ satisfies $r^{L} \leq r \leq r^{U}$.

The upper and lower bound models from Eqs. (1) and (2) will be used in conjunction to derive probabilistic upper and lower bounds on the capacity of the transmission $[1 \rightarrow k+1]$. Denoting the (cumulative) departure process at node $k+1$ by $D(t)$, a probabilistic upper bound on the non-asymptotic (transient) capacity rate is a minimal value $\lambda_{t}^{U}$ such that

$$
\mathbb{P}\left(D(t) \geq \lambda_{t}^{U} t\right) \leq \varepsilon,
$$

where $\varepsilon$ is some fixed violation probability (e.g., $\varepsilon=10^{-3}$ ). A probabilistic lower bound on the non-asymptotic capacity rate is a maximal value $\lambda_{t}^{L}$ such that

$$
\mathbb{P}\left(D(t) \leq \lambda_{t}^{L} t\right) \leq \varepsilon .
$$


We remark that $\lambda_{t}^{U}$ and $\lambda_{t}^{L}$ are non-asymptotic both in time and network size. The behavior of these bounds, relative to multiple levels of burstiness in the arrival process $A(t)$ at the source and especially to the number of hops $k$, is the goal of this paper.

In addition to the arrival model we also need to introduce the service model for single-hop transmissions, which will be instrumental to the derivation of multi-hop results.

\section{B. Service processes}

In order to represent the service of a single-hop transmission, for instance $[1 \rightarrow 2]$, we introduce the (virtual) interfering process $V(t)$, corresponding to node 1 , whose increments $V(t-1, t):=V(t)-V(t-1)$, and $V(0)=0$, are defined for all $t \geq 1$ as [10]

$$
V(t-1, t)=1-X_{1}(t) \prod_{i=2}^{N}\left(1-X_{i}(t)\right) .
$$

Here, $X_{i}(t)$ 's are i.i.d. Bernoulli random variable taking values 1 and 0 , with probabilities $p$ and $1-p$, respectively. Note that as we assumed bursty arrivals at node 1 , it may happen that the multi-access protocol can select node 1 to successfully access the channel $(V(t-1, t)=1)$ but there is nothing to transmit (all the data in $A(t)$ has been transmitted by time $t-1$ ). Due to such situations we emphasize the attribute virtual for the process $V(t)$. The process $V(t)$ is statistically independent of $A(t)$ since $V(t)$ depends only on the corresponding independent Bernoulli random variables.

In the derivations of the lower and upper bounds from Eqs. (3) and (4) we need the MGF and Laplace transforms of $V(t)$, respectively, i.e., for $\theta \neq 0$

$$
E\left[e^{\theta V(t)}\right]=e^{\theta \frac{\log b_{\theta}}{\theta} t}
$$

where $b_{\theta}=1+q\left(e^{\theta}-1\right)$ and $q=1-p(1-p)^{N-1}$. The term $\frac{\log b_{\theta}}{\theta}$ is the relative rate of $V(t)$. The key result in the derivations is the following exact $(\mathrm{min},+)$ representation of the service received by a node in a single-hop transmission.

Theorem 1: (SINGLE-Hop (EXACT) SERVICE REPRESENTATION) Consider the node 1 from Figure 1 and the interfering process $V(t)$ from Eq. (5). Then for all arrival processes $A(t)$ at node 1 , the following equality holds for the departure process $D(t)$ corresponding to single-hop transmissions:

$$
D(t)=\inf _{0 \leq s \leq t}\{A(s)+t-s-V(s, t)\} .
$$

Therefore, the bivariate process

$$
S(s, t)=t-s-V(s, t)
$$

is a stochastic service curve for node 1, i.e.,

$$
D(t)=A * S(t) .
$$

Here, the symbol ' $*$ ' denotes the $(\mathrm{min},+)$ convolution operator defined for all $t \geq 0$ as $A * S(t):=\inf _{0 \leq s \leq t}\{A(s)+S(s, t)\}$. (The proof is omitted; for the lower bound see [10]).

The critical observation in the theorem is that Eqs. (6) and (7) hold for all arrival processes $A(t)$ at node 1 . This invariance of service curves to arrival processes is in fact instrumental in the theory of the network calculus to the derivation of both single and multi-hop queueing results. Concretely, having the service curves at each hop in a multihop scenario, holding for (possibly) unknown input and output processes but directly related from hop to hop, the $(\mathrm{min},+)$ convolution of the service curves provides a characterization of the service received along the entire network path. Using this network path service characterization, multi-hop results can be simply derived using single-hop results [5], [7], [18].

\section{END-TO-END CAPACITY}

In this section we provide the main results of this paper, i.e., lower and upper bounds on the capacity rate along the $[1 \rightarrow$ $2 \rightarrow \cdots \rightarrow k+1]$ path in the network from Figure 1, where the arrival process at node 1 belongs to the EBB class as in Eqs. (1) and (2). We also provide some numerical illustrations of these bounds for the subclass of Markov-Modulated On-Off processes with various levels of burstiness.

Theorem 2: (LOWER AND UPPER BOUNDS ON END-TOEND CAPACITY) Consider the network from Figure 1 described in the previous section. Let the arrival process $A(t)$ at node 1 with relative upper and lower rates $r^{U}$ and $r^{L}$, depending on $\theta>0$, as in Eqs. (1) and (2). If $D(t)$ denotes the departure process at node $k+1$ of the multi-hop transmission $[1 \rightarrow k+1]$, then we have the following probabilistic lower bound on the end-to-end capacity for all $\lambda_{t}^{L}$ and $t \geq 0$

$$
\mathbb{P}\left(D(t) \leq \lambda_{t}^{L} t\right) \leq \inf _{\theta>0, \delta \geq 0,1-\frac{\log b_{\theta}}{\theta}>r^{L}-\delta} M e^{-\theta\left(r^{L}-\delta-\lambda_{t}^{L}\right) t},
$$

where $M=\left(1+\left(\frac{1}{\theta\left(1-\frac{\log b_{\theta}}{\theta}-r^{L}+\delta\right)}\right)^{k}\right), p=\frac{1}{N}, q=1-$ $p(1-p)^{N-1}$, and $b_{\theta}=1+q\left(e^{\theta}-1\right)$. Also, the corresponding upper bound for all $\lambda_{t}^{U}$ and $t \geq 0$ is

$$
\mathbb{P}\left(D(t) \geq \lambda_{t}^{U} t\right) \leq \inf _{\theta>0} e^{-\theta\left(\lambda_{t}^{U}-r^{U}\right)} .
$$

The condition $1-\frac{\log b_{\theta}}{\theta}>r^{L}-\delta$ can be regarded as a stability condition, with the left-hand side term representing the available service rate for a one-hop transmission. The parameter $\delta \geq 0$ is used for optimization: on one hand it relaxes the lower rate $r^{L}$ in the exponential, and on the other hand it enables a bigger optimization space for $\theta$ in the stability condition. This tradeoff results in much tighter bounds than by simply letting $\delta=0$; this rate relaxation technique has been used for similar purposes in [9] and [22].

Proof. Fix $t \geq 0$. According to Theorem 1, the bivariate processes

$$
S_{j}(s, t)=t-s-V_{j}(s, t)
$$

are exact service curve processes for the end-to-end transmission $[1 \rightarrow k+1]$ at each hop $j+1=2,3, \ldots, k+1$. The corresponding interfering processes

$$
V_{j}(s, t)=t-s-\sum_{u=s+1}^{t} X_{1, j}(u) \prod_{i=2}^{N}\left(1-X_{i, j}(u)\right)
$$


are as in Eq. (5) with $X_{i, j}(u)$ being i.i.d. Bernoulli random variables. Applying the convolution theorem in network calculus we have the relationship

$$
D(t)=A * S_{1} * S_{2} * \ldots * S_{k}(t),
$$

coupling the arrival process $A(t)$ at the source node 1 with the departure process $D(t)$ at the destination node $k+1$. Using this relationship we can write for the lower bound, for some $\lambda_{t}^{L} \geq 0$

$$
\begin{gathered}
\mathbb{P}\left(D(t) \leq \lambda_{t}^{L} t\right) \leq \mathbb{P}\left(A * S_{1} * \ldots * S_{k}(t) \leq \lambda_{t}^{L} t\right) \\
\leq \sum_{s=0}^{t} \mathbb{P}\left(A(s)+\inf _{s \leq u_{1} \leq \cdots \leq u_{k-1} \leq t} \sum_{j=1}^{k} S_{j}\left(u_{j-1}, u_{j}\right) \leq \lambda_{t}^{L} t\right) \\
\leq \sum_{s=0}^{t} \mathbb{P}\left(\sup _{s \leq u_{1} \leq \cdots \leq u_{k-1} \leq t}\left(V_{j}\left(u_{j-1}, u_{j}\right)-\left(u_{j}-u_{j-1}\right)\right)\right. \\
\left.\geq A(s)-\lambda_{t}^{L} t\right),
\end{gathered}
$$

where by convention $u_{0}=s$ and $u_{k}=t$. In the second line we applied Boole inequality. Using further repeatedly Boole inequality and the Chernoff bound for some $\theta>0$ we can bound the last sum above by

$$
\begin{aligned}
& \sum_{0 \leq s \leq u_{1} \leq \cdots \leq u_{k-1} \leq t} e^{-\theta r^{L} s} e^{\theta \frac{\log b_{\theta}}{\theta}\left(u_{j}-u_{j-1}\right)} e^{-\theta\left(u_{j}-u_{j-1}\right)} e^{\theta \lambda_{t}^{L} t} \\
= & \sum_{s=0}^{t}\left(\begin{array}{c}
t-s+k-1 \\
k-1
\end{array}\right) e^{-\theta\left(1-\frac{\log b_{\theta}}{\theta}-r^{L}\right)(t-s)} e^{-\theta\left(r^{L}-\lambda_{t}^{L}\right) t},
\end{aligned}
$$

where the binomial term is the number of combinations with repetition. Since the sum is non-increasing in $r^{L}$, we can relax this rate by some $\delta \geq 0$ satisfying the stability condition $1-$ $\frac{\log b_{\theta}}{\theta}>r^{L}-\delta$, and we can further bound the sum by

$$
\sum_{s=0}^{t}\left(\begin{array}{c}
t-s+k-1 \\
k-1
\end{array}\right) e^{-\theta\left(1-\frac{\log b_{\theta}}{\theta}-r^{L}+\delta\right)(t-s)} e^{-\theta\left(r^{L}-\delta-\lambda_{t}^{L}\right) t},
$$

Next we use the equality $\sum_{s \geq 0}\left(\begin{array}{c}s+k-1 \\ k-1\end{array}\right) a^{s}=\left(\frac{1}{1-a}\right)^{k}$ for all $0<a<1$ [14], with $a=e^{-\theta\left(1-\frac{\log b_{\theta}}{\theta}-r^{L}+\delta\right)}$, and the inequality $\left(\frac{1}{1-e^{-x}}\right)^{k} \leq 1+\left(\frac{1}{x}\right)^{k}$ for all $x>0$, and we can bound the last sum by

$$
\mathbb{P}\left(D(t) \leq \lambda_{t}^{L} t\right) \leq M e^{-\theta\left(r^{L}-\delta-\lambda_{t}^{L}\right) t},
$$

with $M$ as in the theorem. Minimizing after $\theta$ and $\delta$ completes the proof for the lower bound.

In turn, for the upper bound, we can write for some $\lambda_{t}^{U} \geq 0$

$$
\begin{gathered}
\mathbb{P}\left(D(t) \geq \lambda_{t}^{U} t\right)=\mathbb{P}\left(A * S_{1} * \ldots * S_{k}(t) \geq \lambda_{t}^{U} t\right) \\
\leq \inf _{0 \leq s \leq u_{1} \leq \cdots \leq u_{k-1} \leq t} \mathbb{P}\left(A(s)+t-s-\sum_{j=1}^{k-1} V_{j}\left(u_{j-1}, u_{j}\right)\right. \\
\left.\geq \lambda_{t}^{U} t\right) \\
\leq \inf _{0 \leq s \leq t} e^{\theta\left(1+\frac{\log b_{-\theta}}{\theta}-r^{U}\right)(t-s)} e^{-\theta\left(\lambda_{t}^{U}-r^{U}\right)} .
\end{gathered}
$$

The infimum is attained at $s=t$. Minimizing over $\theta$ completes the proof.

For comparison we next give the non-asymptotic capacity results in the network from Figure 1 where node 1 is always saturated. A probabilistic lower bound on the capacity rate is for all $t, \tilde{\lambda}_{t}^{L} \geq 0$

$$
\mathbb{P}\left(D(t) \leq \tilde{\lambda}_{t}^{L} t\right) \leq \inf _{\theta>0}\left(\begin{array}{c}
t+k-2 \\
k-1
\end{array}\right) e^{-\theta\left(1-\frac{\log b_{\theta}}{\theta}-\tilde{\lambda}_{t}^{L}\right) t} .
$$

A corresponding upper bound is

$$
\mathbb{P}\left(D(t) \geq \tilde{\lambda}_{t}^{U} t\right) \leq \inf _{\theta>0} e^{-\theta\left(1+\frac{\log b_{-\theta}}{\theta}-\tilde{\lambda}_{t}^{U}\right) t},
$$

where $b_{\theta}$ is defined as in Theorem 2 . The proof of these bounds follows along the lines of the proof of Theorem 2; the key difference is that in the case when node 1 is always saturated one has $A * S_{1} * \ldots * S_{k}(t)=S_{1} * \ldots * S_{k}(t)$.

For some fixed violation probability $\varepsilon$ all the four asymptotic bounds (i.e., $\lambda_{t}^{L}, \lambda_{t}^{U}, \tilde{\lambda}_{t}^{L}$, and $\tilde{\lambda}_{t}^{U}$, with $t \rightarrow \infty$ ) scale as $\Theta(1)$ in the number of hops $k$. However, at a fixed time scale $t$, the (non-asymptotic) lower bounds for both unsaturated and saturated regimes scale as

$$
\lambda_{t}^{L}, \tilde{\lambda}_{t}^{L}=\Omega(-k),
$$

i.e., they decrease linearly in $k$. Since the non-asymptotic endto-end capacity rate decreases at least linearly in $k$, the two lower bounds from Eqs. (8) and (11) capture the right scaling, i.e., $\Theta(-k)$.

In turn,the corresponding (non-asymptotic) upper bounds from Eqs. (9) and (12) scale as

$$
\lambda_{t}^{U}, \tilde{\lambda}_{t}^{U}=\mathcal{O}(1),
$$

which are clearly asymptotically loose. The technical reason lies in the evaluation of sample bounds using the argument from the second line of Eq. (10), which does not capture the decay in $k$. We note that the underlying bound

$$
\mathbb{P}\left(\inf _{s} X_{s} \geq \sigma\right) \leq \inf _{s} \mathbb{P}\left(X_{s} \geq \sigma\right),
$$

for some stochastic process $X_{s}$, has been frequently used to provide lower bounds on the tail probability for queue overflow (see for instance [23], [8], [3]). This (rather trivial) bound has been argued to be reasonably accurate based on the existence of a dominant time scale in queueing analysis. However, for the end-to-end scenario herein, this dominant time scale argument is clearly inapplicable. Asymptotically tight upper bounds in $k$ remains an open problem.

It is also open whether the $\Theta(-k)$ scaling holds for other types of arrivals, such as heavy-tailed and self-similar, or other types of network models which may create subtle correlations between adjacent hops possibly leading to super-linear scaling decay of the end-to-end (non-asymptotic) capacity rate; such behavior has been observed in wired networks with packetized EBB traffic, where end-to-end delays, and hence end-to-end (non-asymptotic) capacity rates as well, were observed to scale super-linearly [6]. 


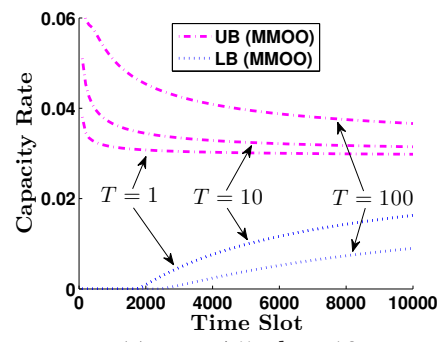

(a) $u=.75, k=10$

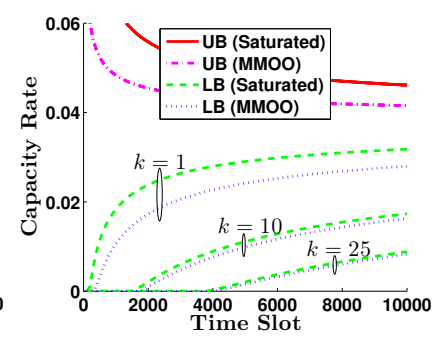

(b) $u=.99, T=10$
Fig. 2. Non-asymptotic capacity rates of the transmission $[1 \rightarrow k+1]$ in the network from Figure 1 for different utilization factors $u$, burstiness parameter $T=\frac{1}{\lambda}+\frac{1}{\mu}$, and number of hops $k\left(\varepsilon=10^{-3}\right)$

For brief numerical illustrations we consider the case of a Markov Modulated On-Off (MMOO) process, driven by a Markov process with two states 'On' and 'Off' and transition rates $\lambda$ and $\mu$ between the two, peak rate $P$ while in the 'On' state, and burstiness parameter $T=\frac{1}{\lambda}+\frac{1}{\mu}$ representing the average time for the chain to change states twice. Explicit expressions for $r^{U}$ and $r^{L}$ in Eqs. (1) and (2) are available in [11].

For the network from Figure 1 we consider the following numerical settings: the number of nodes within interference ranges is $N=10$, the number of hops is $k=10$, and the input traffic parameters are properly scaled such that the utilization factor at the first hop is $u=.75$. Also, the peak rate is twice as much as the average input rate, and the violation probability is $\varepsilon=10^{-3}$.

Figure 2.(a) illustrates the behavior of the upper (UB) and lower (LB) bounds (i.e., from Eqs. (9) and (8)) on the nonasymptotic capacity rate of the transmission $[1 \rightarrow k+1]$ in the network from Figure 1 with respect to multiple levels of burstiness $(T=1,10,100)$. The figure shows in particular that the capacity rates are eventually sensitive to burstiness, i.e., burstiness eventually dominates the effects of multi-access. One such effect concerns the situation in which multi-access may slow down the end-to-end output of a bursty source, for a sufficiently long time interval, and which may appear similar as the output of a less bustier source, for the same time interval. We point out that our analysis, and consequently the illustrated bounds, fully account for the unknown burstiness at the relay nodes.

In Figure 2.(b) we show the same bounds as in (a) but at utilization $u=.99$, and additionally the upper and lower bounds for the saturated case from Eqs. (12) and (11). The figure illustrates the decay of the lower bounds for different number of hops $k=1,10,25$, and indicates that at least in this moderate range the value of $k$ is not the dominant factor, as the bounds appear to decay slower than the linear theoretical prediction.

\section{CONCLUSIONS}

In this paper we have derived explicit non-asymptotic capacity rates in terms of both upper and lower bound on their distributions. The main novelty of the bounds is that they were obtained under a burstiness constraint at the data source, and a large traffic class (EBB) has been considered. The value of the obtained results is that they provide immediate insight on how the network capacity scales depending on practical parameters such as the degree of burstiness, the network size, or the time scales. The paper opens the question whether such nonasymptotic capacity results are further influenced by subtle correlations which may arise due to burstiness patterns and/or network topology.

\section{REFERENCES}

[1] N. Abramson. The throughput of packet broadcasting channels. IEEE Transactions on Communications, 25(1):117 - 128, 1977.

[2] I. Akyildiz and X. Wang. Wireless Mesh Networks. Wiley, 2009.

[3] R. Boorstyn, A. Burchard, J. Liebeherr, and C. Oottamakorn. Statistical service assurances for traffic scheduling algorithms. IEEE Journal on Selected Areas in Communications Special Issue on Internet QoS, 18(12):2651-2664, Dec. 2000.

[4] C. Bordenave, D. McDonald, and A. Proutiere. Performance of random medium access control, an asymptotic approach. In ACM Sigmetrics, pages 1-12, 2008 .

[5] J.-Y. Le Boudec and P. Thiran. Network Calculus. Springer Verlag, Lecture Notes in Computer Science, LNCS 2050, 2001.

[6] A. Burchard, J. Liebeherr, and F. Ciucu. On superlinear scaling of network delays. to appear in IEEE/ACM Transactions on Networking.

[7] C.-S. Chang. Performance Guarantees in Communication Networks. Springer Verlag, 2000.

[8] J. Choe and N. B. Shroff. A central-limit-theorem-based approach for analyzing queue behavior in high-speed networks. IEEE/ACM Transactions on Networking, 6(5):659-671, Oct. 1998.

[9] F. Ciucu, A. Burchard, and J. Liebeherr. Scaling properties of statistical end-to-end bounds in the network calculus. IEEE Transactions on Information Theory, 52(6):2300-2312, June 2006.

[10] F. Ciucu, O. Hohlfeld, and P. Hui. Non-asymptotic throughput and delay distributions in multi-hop wireless networks. In Allerton Conference on Communications, Control and Computating, 2010.

[11] C. Courcoubetis and R. Weber. Buffer overflow asymptotics for a buffer handling many traffic sources. Journal of Applied Probability, 33(3):886-903, Sept. 1996.

[12] R. Cruz. A calculus for network delay, parts I and II. IEEE Transactions on Information Theory, 37(1):114-141, Jan. 1991.

[13] A. Ephremides and B. E. Hajek. Information theory and communication networks: An unconsummated union. IEEE Transactions on Information Theory, 44(6):2416-2434, Oct. 1998.

[14] M. Fidler. An end-to-end probabilistic network calculus with moment generating functions. In IEEE International Workshop on Quality of Service (IWQoS), pages 261-270, 2006.

[15] R. G. Gallager. A perspective on multiaccess channels. IEEE Transactions on Information Theory, 31(2):124-142, Mar. 1985.

[16] Y. Gao, D.-M. Chiu, and J. C. S. Lui. Determining the end-to-end throughput capacity in multi-hop networks: methodology and applications. In ACM Sigmetrics/Performance, pages 39-50, June 2006.

[17] P. Gupta and P. R. Kumar. The capacity of wireless networks. IEEE Transactions on Information Theory, 46(2):388-404, Mar. 2000.

[18] Y. Jiang and Y. Liu. Stochastic Network Calculus. Springer, 2008.

[19] L. Kleinrock. Queueing Systems, volume 1. John Wiley and Sons, 1975.

[20] L. Kleinrock and J. Silvester. Optimum transmission radii for packet radio networks or why six is a magic number. In Proceedings of IEEE National Telecommunication Conference, pages 4.3.1-4.3.5, 1978.

[21] L. Kleinrock and F. A. Tobagi. Packet switching in radio channels: Part I-carrier sense multiple-access modes and their throughput-delay characteristics. IEEE Transactions on Communications, 23(12):14001416, Dec. 1975

[22] J. Liebeherr, A. Burchard, and F. Ciucu. Non-asymptotic delay bounds for networks with heavy-tailed traffic. In IEEE Infocom, 2010.

[23] M. Montgomery and G. de Veciana. On the relevance of time scales in performance oriented traffic characterizations. In IEEE Infocom, pages 513-520, 1996.

[24] O. Yaron and M. Sidi. Performance and stability of communication networks via robust exponential bounds. IEEE/ACM Transactions on Networking, 1(3):372-385, June 1993. 